\title{
Flute Mode Turbulence and Related Transport in the Divertor of a Mirror
}

\author{
Isao KATANUMA, Hiroshi SAIMARU, Yoshinari MIZOGUCHI, Katsuya YASHIRO and \\ Vladimir P. PASTUKHOV ${ }^{1)}$ \\ Plasma Research Center, University of Tsukuba, Tsukuba 305-8577, Japan \\ ${ }^{1)}$ RRC Kurchatov Institute, Kurchatov Square, 1, 123182 Moscow, Russia
}

(Received 23 October 2007 / Accepted 21 January 2008)

\begin{abstract}
A computer code, which was written to investigate flute modes and related transport, can be applied to a magnetic shearless confinement system as well as a tandem mirror. Computer simulation conducted in a modeled magnetic divertor with initial rigid plasma rotation shows that flute modes enhance radial transport during its growing phase, and shear (zonal) flows appear in the final stage.
\end{abstract}

(c) 2008 The Japan Society of Plasma Science and Nuclear Fusion Research

Keywords: divertor, flute, interchange, simulation, mirror

DOI: $10.1585 /$ pfr.3.S1072

\section{Introduction}

A magnetic divertor is an application of the stable dipole magnetic configuration of a planet. Its configuration is in equilibrium up to $\beta \simeq 1$, where $\beta$ is the ratio of plasma pressure to the vacuum magnetic field on axis. An open magnetic confinement system such as GAMMA10 can form a fully axisymmetric system with MHD stable state by containing a magnetic divertor region [1].

A typical divertor magnetic field is shown in Fig. 1, where the axial length $L=200$, and a magnetic null point is located at $(r, z)=(65,0)$. This axisymmetric mirror plasma is found to be stabilized by plasma compressibility rather than by the ion finite Larmor radius effects around the magnetic null for denser radial density profiles [2]. That is, $\partial p U^{\gamma} / \partial \psi>0$ is the stability condition of plasma in Fig. 1, where $p$ is plasma pressure, $U \equiv \int \mathrm{d} \ell / B$ is the specific volume of a magnetic field line, $\gamma$ is the heat index and $2 \pi \psi$ is the magnetic flux surrounded by the surface $\psi=$ const.

Flute modes are one of the most dangerous instabilities in the magnetic shearless confinement system; therefore stability analysis of the flute modes usually has a priority over that of other modes [3]. However the transport process resulting from the flute instabilities has not been studied in detail to the authors' knowledge. Thus, the purpose of this paper is to investigate the flute instability and related plasma radial transport in the magnetic divertor shown in Fig. 1.

As mentioned above in this paper, the flute modes are stabilized mainly by plasma compressibility in a divertor mirror cell. Therefore, the fluid approximation can be applied to the flute mode analysis. In the marginally stable state, the radial profile of plasma pressure is assumed to
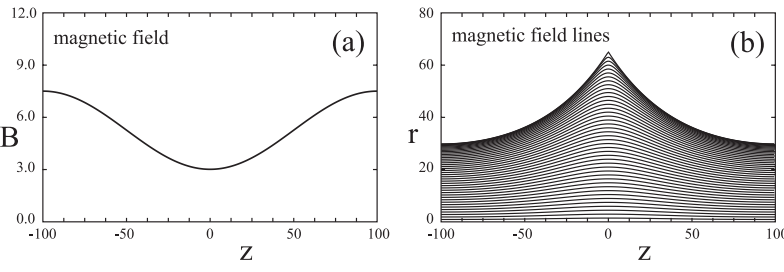

Fig. 1 Modeled magnetic divertor. (a) is the axial profile of magnetic field and (b) plots magnetic field lines.

satisfy the relation $p U^{\gamma}=$ const. Ions passing near the magnetic null region do not conserve their magnetic moment $\mu$, which can disturb the marginally stable state; i.e., $\partial p U^{\gamma} / \partial \psi \lesssim 0$, where the flute modes become unstable. In the following, we consider the slightly unstable state of the flute modes.

\section{Basic Equation}

The degrees of freedom of compressive and shear Alfvén modes and acoustic modes are not required to be considered to calculate the flute instability, because those modes are more stable than the flute modes, and those modes usually have high frequency oscillations. One of authors (Pastukhov [4]) has proposed a method to remove the Alfvén and acoustic modes from the MHD equations. Thus, the ideal MHD equation is obtained by taking a variation of Lagrangian with respect to $\boldsymbol{\xi}$, where the plasma displacement $\boldsymbol{\xi}$ is arbitrarily chosen. In order to remove unnecessary degree of freedom, the restrictions $\nabla \cdot(\xi / U)=0$ and $\nabla \times(\boldsymbol{\xi} \times \boldsymbol{B})=0$ were added to the Lagrangian. The resultant equation of motion is given as 


$$
\begin{aligned}
\frac{\partial \hat{w}}{\partial t}+ & \llbracket \Phi, \hat{w} \rrbracket-\llbracket \hat{\rho},\left\langle\frac{v_{\alpha}^{2}}{2}\right\rangle \rrbracket+\frac{1}{U^{\gamma}} \frac{\partial U}{\partial \psi} \frac{\partial\left(\hat{\rho}_{0} \tilde{T}+\hat{T}_{0} \tilde{\rho}\right)}{\partial \varphi} \\
= & U Q_{w}^{*} \\
& +\frac{3 X_{M}}{20}\left(\frac{T_{\mathrm{i}}+T_{\mathrm{e}}}{2 T_{\mathrm{i}}}\right)\left\{\frac{\partial}{\partial \psi}\left(\hat{\rho}\left\langle r^{2}\right\rangle \frac{\partial}{\partial \psi}\left(\frac{1}{U^{2 / 3} \sqrt{\hat{T}_{0}}} \frac{a^{2}}{\left\langle r^{2}\right\rangle} \hat{w}\right)\right)\right. \\
& \left.+\frac{a^{2}}{\left\langle r^{2}\right\rangle}\left\langle\frac{1}{r^{2} B^{2}}+\lambda^{2} B^{2}\right\rangle \frac{\hat{\rho}}{U^{2 / 3} \sqrt{\hat{T}_{0}}} \frac{\partial^{2} \hat{w}}{\partial \varphi^{2}}\right\} .
\end{aligned}
$$

Here $a^{2}=\left\langle r^{4}\right\rangle /\left\langle r^{2}\right\rangle$, and $\hat{w}$ has the following form.

$$
\hat{w}=\frac{\partial}{\partial \psi}\left(\hat{\rho}\left\langle r^{2}\right\rangle \frac{\partial \Phi}{\partial \psi}\right)+\frac{\partial}{\partial \varphi}\left(\hat{\rho}\left\langle\frac{1}{r^{2} B^{2}}+\lambda^{2} B^{2}\right\rangle \frac{\partial \Phi}{\partial \varphi}\right),
$$

where $\Phi \equiv c \phi ; c$, light speed; $\phi$, electrostatic potential; $w$ is related to the specific volume averaged vorticity $\nabla \times$ $\left(\rho c \boldsymbol{B} \times \nabla \phi / B^{2}\right)$ due to plasma $\boldsymbol{E} \times \boldsymbol{B}$ drift flux; and $v_{\alpha}^{2}$ is the square of plasma fluid velocity. Poisson bracket is defined as

$$
\llbracket \Phi, \hat{w} \rrbracket \equiv \frac{\partial \Phi}{\partial \psi} \frac{\partial \hat{w}}{\partial \varphi}-\frac{\partial \Phi}{\partial \varphi} \frac{\partial \hat{w}}{\partial \psi} .
$$

The second term in the right hand side in Eq. (1) represents the viscosity of fluid, and the third term contains the effects by which the vorticity can move along a magnetic null.

The transport equations of mass density and heat are given in the following equation on the assumption of the adiabatic index $\gamma=5 / 3$.

$$
\frac{\partial \hat{\rho}_{0}}{\partial t}-\frac{\partial}{\partial \psi}\left(\tilde{\rho} \frac{\overline{\partial \Phi}}{\partial \varphi}\right)=4 \pi d_{M} \frac{\partial}{\partial \psi}\left[\frac{\hat{\rho}_{0}\left\langle r^{2}\right\rangle U}{\hat{T}_{0}^{3 / 2}} \frac{\partial}{\partial \psi}\left(\frac{\hat{\rho}_{0} \hat{T}_{0}}{U^{5 / 3}}\right)\right]+\bar{Q}_{\rho}^{*} U
$$

$\frac{\partial \tilde{\rho}}{\partial t}+\llbracket \Phi, \tilde{\rho} \rrbracket+\frac{\partial}{\partial \psi}\left(\tilde{\rho} \frac{\overline{\partial \Phi}}{\partial \varphi}\right)-\frac{\partial \Phi}{\partial \varphi} \frac{\partial \hat{\rho}_{0}}{\partial \psi}=\tilde{Q}_{\rho}^{*} U+4 \pi d_{M}$

$\times\left(\frac{\partial}{\partial \psi}\left[\frac{\hat{\rho}_{0}\left\langle r^{2}\right\rangle U}{\hat{T}_{0}^{3 / 2}} \frac{\partial}{\partial \psi}\left(\frac{\tilde{\rho} \hat{T}_{0}}{U^{5 / 3}}\right)\right]+\left[\frac{\hat{\rho}_{0}}{\hat{T}_{0}^{1 / 2} U^{2 / 3}}\left\langle\frac{1}{r^{2} B^{2}}\right\rangle \frac{\partial^{2} \tilde{\rho}}{\partial \varphi^{2}}\right]\right)$,

$$
\begin{array}{r}
\frac{\partial \hat{T}_{0}}{\partial t}-\frac{\partial}{\partial \psi}\left(\tilde{T} \frac{\overline{\partial \Phi}}{\partial \varphi}\right)=X_{M} \frac{2 U^{2 / 3}}{3 \hat{\rho}_{0}} \frac{\partial}{\partial \psi}\left(\frac{\hat{\rho}_{0}^{2}\left\langle r^{2}\right\rangle}{U} \frac{\partial}{\partial \psi}\left(\frac{\sqrt{\hat{T}_{0}}}{U^{1 / 3}}\right)\right) \\
+\frac{8 \pi d_{M}}{3 \sqrt{\hat{T}_{0}}} \frac{\partial}{\partial \psi}\left(\left\langle r^{2}\right\rangle U \frac{\partial}{\partial \psi}\left(\frac{\hat{\rho}_{0} \hat{T}_{0}}{U^{5 / 3}}\right)\right)+\frac{U^{5 / 3}}{\hat{\rho}} \bar{Q}_{T}^{*}
\end{array}
$$

$\frac{\partial \tilde{T}}{\partial t}+\llbracket \Phi, \tilde{T} \rrbracket+\frac{\partial}{\partial \psi}\left(\tilde{T} \frac{\overline{\partial \Phi}}{\partial \varphi}\right)-\frac{\partial \Phi}{\partial \varphi} \frac{\partial \hat{T}_{0}}{\partial \psi}=\frac{U^{5 / 3}}{\hat{\rho}} \tilde{Q}_{T}^{*}+X_{M} \frac{U^{2 / 3}}{3 \hat{\rho}_{0}}$ $\times \frac{\partial}{\partial \psi}\left(\frac{\hat{\rho}_{0}^{2}\left\langle r^{2}\right\rangle}{U} \frac{\partial}{\partial \psi}\left(\frac{\tilde{T}}{U^{1 / 3} \sqrt{\hat{T}_{0}}}\right)\right)+\frac{X_{M} \hat{\rho}_{0}}{3 U^{2 / 3} \sqrt{\hat{T}_{0}}}\left\langle\frac{1}{r^{2} B^{2}}\right\rangle \frac{\partial^{2} \tilde{T}}{\partial \varphi^{2}}$.

Here $\rho \equiv M_{\mathrm{i}} n_{\mathrm{i}}+M_{\mathrm{e}} n_{\mathrm{e}}=\langle\rho\rangle \equiv \hat{\rho} / U$ is mass density. The slow time variable equilibrium component $\hat{\rho}_{0}\left(\epsilon^{3} t, \psi\right)$ and fast variable fluctuating components $\tilde{\rho}(\epsilon t, \psi, \varphi)$ are defined, where the equilibrium and fluctuating quantities are represented by the hat with subscript $\hat{}_{0}$ and the tilde $\sim$, respectively, and $\hat{\rho}=\hat{\rho}_{0}+\tilde{\rho}$. The quantity $\hat{T}$ is related to the temperature defined by $\hat{T} \equiv p U^{\gamma} / \hat{\rho}=\left(T_{\mathrm{i}}+T_{\mathrm{e}}\right) U^{\gamma-1} / M_{\mathrm{i}}$.
The symbol $\bar{A}$ indicated the average of $A$ over $\varphi, \hat{A}$ indicates the quantity of $A$ integrated in the specific volume of a magnetic field line, and $\langle A\rangle$ indicates the integration of $A$ along a magnetic field line,

$\bar{A} \equiv \frac{1}{2 \pi} \int_{0}^{2 \pi} A \mathrm{~d} \varphi, \quad \hat{A} \equiv \int \frac{A \mathrm{~d} \zeta}{J(\psi, \varphi, \zeta)}, \quad\langle A\rangle \equiv \frac{\hat{A}}{U}$,

where $J(\psi, \varphi, \zeta) \equiv \nabla \psi \times \nabla \varphi \cdot \nabla \zeta$ is Jacobian. The magnetic field line curvatures are included in the coefficients $U,\left\langle r^{2}\right\rangle$, $\left\langle 1 / r^{2} B^{2}\right\rangle$ in the Eqs. (1)-(7), and the definition of $U$ is

$$
U \equiv \int \frac{\mathrm{d} \zeta}{J(\psi, \varphi, \zeta)} .
$$

The coordinates $(\psi, \varphi, \zeta)$ adopted here are the flux coordinates, where the magnetic field is represented as $\boldsymbol{B}=$ $\nabla \psi \times \nabla \varphi$. Here, $2 \pi \psi$ is the magnetic flux inside the surface of $\psi=$ const., and $\varphi$ corresponds to an angle coordinate. The remaining coordinate $\zeta$ is usually taken as the $z$-axis or along a magnetic field line.

The classical diffusions included in Eqs. (1)-(7) is defined by

$$
\begin{aligned}
& X_{M}=\chi_{\perp} \frac{B^{2}}{\rho}\left(\frac{4 T_{\mathrm{i}}}{T_{\mathrm{i}}+T_{\mathrm{e}}}\right)\left(\frac{p}{\rho}\right)^{1 / 2}, \\
& d_{M}=\frac{m_{\mathrm{e}}^{1 / 2}\left(T_{\mathrm{i}}+T_{\mathrm{e}}\right) T_{\mathrm{i}}^{1 / 2}}{\sqrt{2} m_{\mathrm{i}}^{1 / 2} T_{\mathrm{e}}^{3 / 2}}\left(\frac{B^{2}}{4 \pi \rho}\left(\frac{p}{\rho}\right)^{1 / 2} \chi_{\perp}\right), \\
& \chi_{\perp}=\frac{T_{\mathrm{i}}}{m_{\mathrm{i}} \omega_{\mathrm{ci}}^{2} \tau_{\mathrm{i}}}, \quad \epsilon^{3} \equiv \frac{\chi_{\perp}}{b c_{\mathrm{s}}}\left(\frac{2 T_{\mathrm{i}}}{T_{\mathrm{i}}+T_{\mathrm{e}}}\right) .
\end{aligned}
$$

Here $\tau_{\mathrm{i}}$ is the classical ion-ion coulomb collision time [5], $c_{\mathrm{s}} \equiv \sqrt{\gamma p / \rho}$ is the ion sound speed, and $b$ is a distance defined by $b \equiv \sqrt{\psi_{b} / B_{M}}$, where the subscript ${ }_{M}$ indicates some axial position on the axis. Here $\psi_{b}$ is the flux coordinate of the magnetic null point. The quantities related to classical transport $X_{M}$ and $d_{M}$ in Eq. (10) are the dimensionless quantities that are constant along a magnetic field line. The mass density $\rho$ and other plasma quantities $T_{\mathrm{e}}$ and $T_{\mathrm{i}}$ are assumed to be constant along a magnetic field line throughout this paper. The parameter $\epsilon$ defined in Eq. (11) is a small expansion parameter, where we assume $\epsilon^{2}=10^{-2}$ in the numerical calculation presented in the next section in this paper.

The basic equations in this section contain the interchange modes (similar to the Rayleigh-Taylor instabilities) and the modes associated with the presence of nonuniform plasma flows (similar to the Kelvin-Helmholtz instabilities) as well as the electrostatically incompressible stable plasma flows. Thus, this close set of equations describe the nonlinear low-frequency MHD plasma convection and resulting transport processes in weakly dissipative plasmas in axisymmetric shearless systems.

\section{Numerical Results}

Numerical calculation using the basic equations (1)(7) is conducted in the magnetic divertor shown in Fig. 1 
(a)

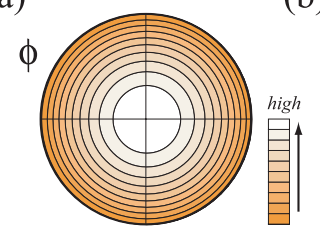

(b)

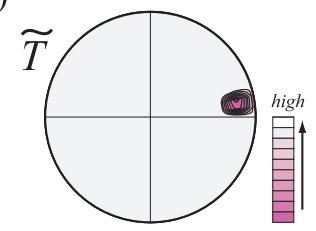

Fig. 2 Initial condition of $\phi$ and $\tilde{T}$.

for the purpose of investigating the effects of flute mode fluctuations on the plasma radial transport.

The effects that fluid can flow freely in the azimuthal direction along a magnetic null line are included through $\left\langle\lambda^{2} B^{2}\right\rangle$ in Eqs. (1) and (2). The potential $\Phi$ is determined using Eq. (2). A dimensionless parameter $\epsilon$ in Eq. (11) contains the magnitude of the classical diffusion.

The equations (1)-(7) have steady state solutions if the classical diffusion is neglected, i.e., $\epsilon=0$, that is

$\hat{\rho}_{0}(\psi)=$ const., $\quad \hat{T}_{0}(\psi)=$ const.,$\quad \hat{w}_{0}(\psi)=$ const.

The field line integrated vorticity $w_{0}=-1$ provides plasma azimuthal rigid rotation, so that there is no shear flow in this initial condition. As long as Eq. (12) is satisfied, no fluctuations are generated in Eqs.(1)-(7). Therefore, we add a small fluctuation $\tilde{T}$ to the initial condition Eq. (12), i.e., $\hat{\rho}_{0}=1, \hat{T}_{0}=1$, and $\hat{w}_{0}=-1$, in the following numerical calculation, where the initial perturbation $\tilde{T}$ added to equilibrium temperature $\hat{T}_{0}$ is plotted in Fig. 2 (b).

The region to be calculated is $\psi<\psi_{b}$ and $-100<$ $z<100$, as plotted in Fig. 1. The external source terms are assumed to be zero, i.e., $Q_{w}^{*}=\bar{Q}_{p}^{*}=\bar{Q}_{T}^{*}=0$ and $\tilde{Q}_{p}^{*}=$ $\tilde{Q}_{T}^{*}=0$ in Eqs. (1)-(7). The boundary conditions adopted here are that $\partial \tilde{\rho} / \partial x=0$ and $\partial \tilde{T} / \partial x=0$ at $x=1$, and the inward flow of vortex $\hat{w}$ is set to be zero at $x=1$. Here, $x$ is a normalized radial coordinate defined by $x \equiv \sqrt{\psi / \psi_{b}}$

Henceforth, the normalized time $\tau$ defined as $\tau=$ $\left(\epsilon c_{\mathrm{S} M} / b\right) t$ is introduced. The specific volume averaged perturbed quantity $\widetilde{A}$ is plotted in place of $\tilde{A}$ in the following figures. The initial condition $\hat{w}_{0}=-1$ gives the axisymmetric potential profile shown in Fig. 2 (a), where the plasma rotates clockwise rigidly around the axis by $2 \pi$ during $\tau \simeq 4$. This initial condition is unstable for the flute modes.

The flute modes grow in a linear phase with the rotation of plasma around the axis by $\boldsymbol{E} \times \boldsymbol{B}$-drift, and then the first big flute instability appears at $\tau=12$ in Fig. 3. A typical parasol shaped equi-contour surfaces of RayleighTaylor instabilities at $\widetilde{T} \equiv \tilde{T} / U^{2 / 3}$ can be seen in Fig. 3 (d).

The radial profiles and fluxes of mass density $\left(\Gamma_{\alpha}\right)$ and temperature $\left(\Gamma_{T}\right)$ are plotted at $\tau=12$ in Fig. 4. Initial flux volume integrated mass density $\hat{\rho}_{0}=\rho_{0} U$ and temperature $\hat{T}_{0}=T_{0} U^{2 / 3}$ profiles are similar to the initial profiles, excluding the region $x \simeq 1$ where the classical radial flux dominates, as shown in Figs. 4 (e) and (f). In time $=\mathbf{1 2 . 0}$

(a)

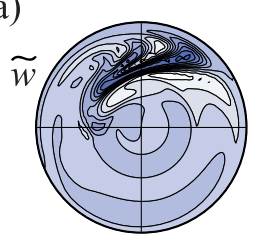

(b)

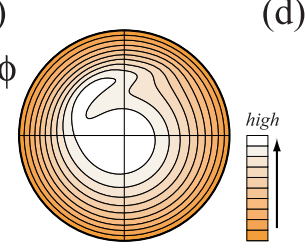

(c)

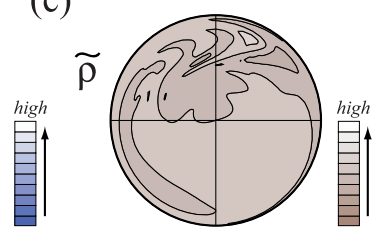

(d)

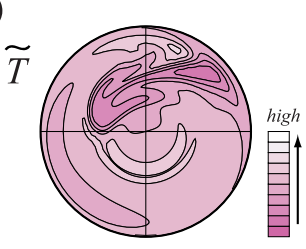

Fig. 3 The potential and the perturbed quantities at $\tau=12$.

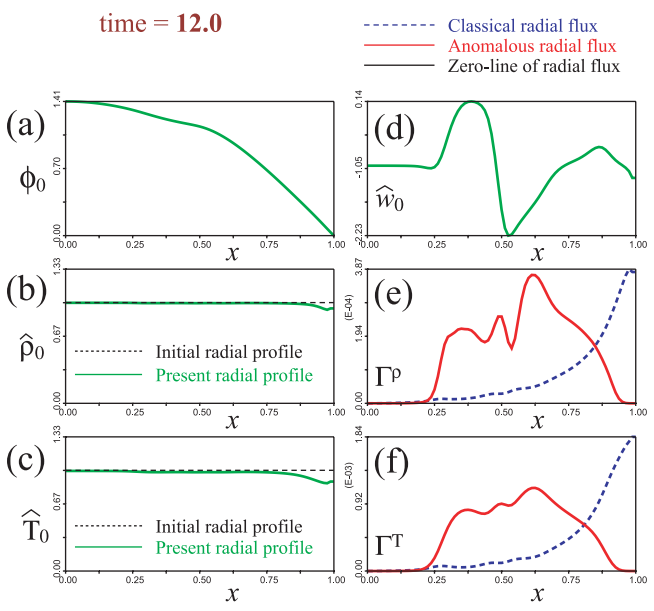

Fig. 4 Radial profiles of $\phi_{0}, \hat{\rho}_{0}, \hat{T}_{0}$, and $\hat{w}_{0}$, and radial fluxes of mass density $\Gamma_{\rho}$ and temperature $\Gamma_{T}$ at $\tau=12$.

the region where the flute instability occurs, the anomalous radial fluxes are much larger than the classical ones, as shown in Figs. 4 (e) and (f). The plasma rotates clockwise in whole region, as shown in Fig. 4 (b), where the potential has a monotonously decreasing radial profile. In the region $x \lesssim 0.25$, where the flute modes do not reach yet, classical transport dominates and plasma rotates rigidly in the azimuthal direction. The parasol shaped low temperature region generated by the flute instability in Fig. 3 continues at $\tau \simeq 10$ and then disappears.

The flute instabilities appear and disappear repeatedly in time with increasing radial transport during the existence of the instabilities. Figure 5 plots the profiles of perturbation quantities at $\tau=44$. The potential has a peak off-axis at that time, as shown in Fig. 5 (b). The low temperature region penetrates the axis, as shown in Fig. 5 (d), which is accompanied by the penetration of the low density region, as shown in Fig. 5 (c). Many short wavelength perturbations are seen in mass density $\widetilde{\rho}$ than that in temperature $\widetilde{T}$, because the modes associated with the presence of nonuniform plasma flows (similar to the Kelvin- 
time $=44.0$

(a)

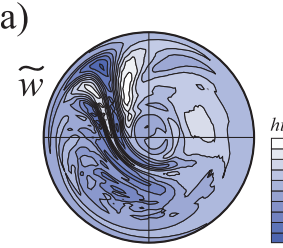

(b)

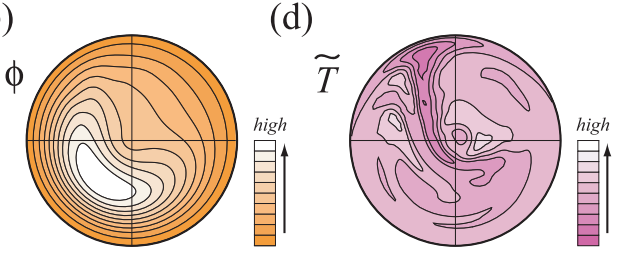

(c)

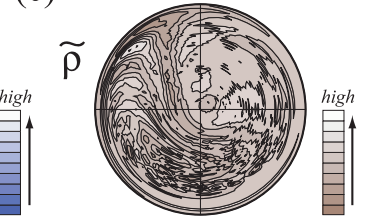

(d)
Fig. 5 Potential and perturbed quantities at $t=44$.
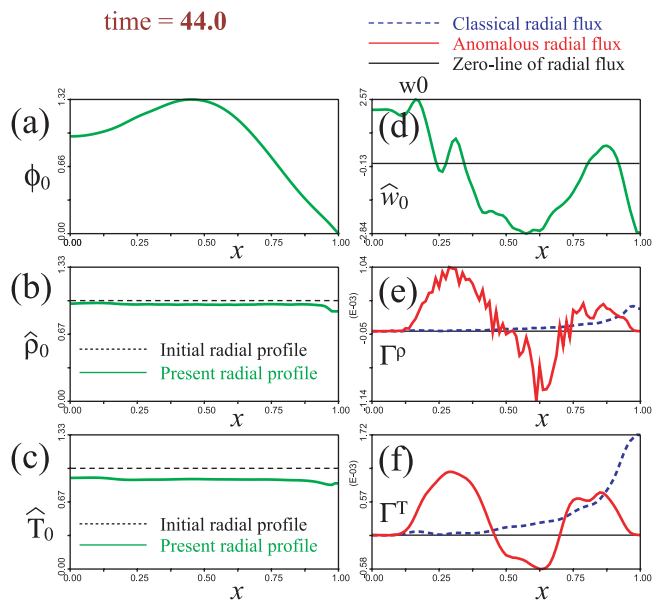

Fig. 6 Radial profiles of $\phi_{0}, \hat{\rho}_{0}, \hat{T}_{0}$, and $\hat{w}_{0}$, and radial fluxes of mass density $\Gamma_{\rho}$ and temperature $\Gamma_{T}$ at $\tau=44$.

Helmholtz instabilities) grow in mass density where the classical diffusion coefficient of mass density in Eq. (5) is much smaller than that of the temperature in Eq. (7).

The radial profile of potential $\phi_{0}$ has a maximum at $x \simeq 0.45$ at $\tau=44$ in Fig. 6 (a). That is, two counter flows exist at this time. Fig. 6(d) plots the radial profile of $w_{0}$, maximum and minimum values of which are 2.57 and -2.87 , respectively. These magnitudes are larger than the initial magnitude of $w_{0}$. Because the total vorticity is conserved in this simulation, there is inward transport of angular momentum. In the region $0.1<x<0.8$, the enhanced radial transport exists and the plasma oscillates radially as shown in Figs. 6 (e) and (f).

The system comes to a quasi-steady state after the long run, as shown in Fig. 7; i.e., the structures do not change much with time. In this state, no perturbations are observed in the region $x \lesssim 0.6$ in Fig. 7. Although the potential in Fig. 7 (b) has a nearly axisymmetric profile, its radial profile is not constant, as shown in Fig. 8 (a). The radial profile $w_{0}$ is also not constant radially, which indicates that there are shear flows (not rigid rotation).

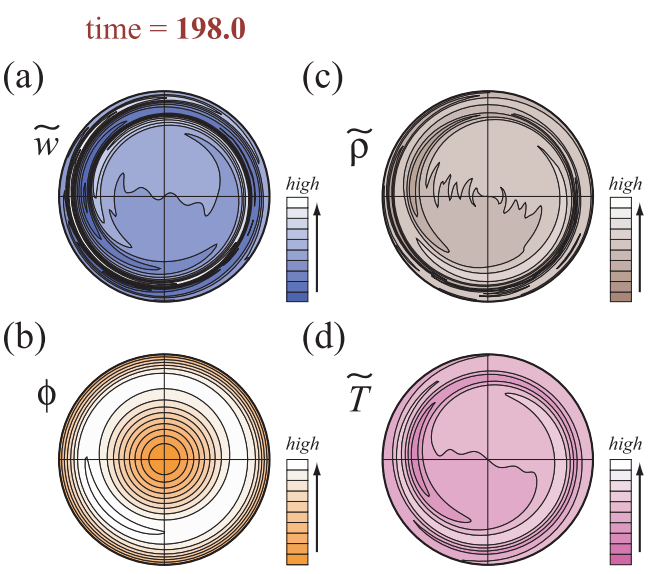

Fig. 7 Potential and perturbed quantities at $t=198$.

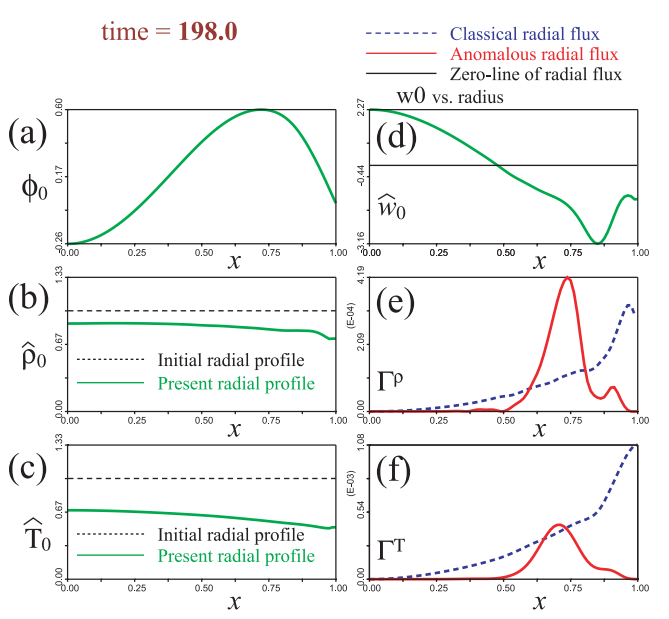

Fig. 8 Radial profiles of $\phi_{0}, \hat{\rho}_{0}, \hat{T}_{0}$, and $\hat{w}_{0}$ and radial fluxes of mass density $\Gamma_{\rho}$ and temperature $\Gamma_{T}$ at $\tau=198$.

In the region $x \gtrsim 0.6$ in Fig. 7 , the vortex structures of $\widetilde{w}, \widetilde{\rho}$, and $\widetilde{T}$ are seen, where the anomalous radial transports are enhanced in $\rho_{0}$ and $T_{0}$ in Figs. 8 (e) and (f). After the vortex $\hat{w}$ in the peripheral region was carried in the core region by the flute instabilities, as plotted in Figs. 3 (a) and 5 (a), its magnitude increased by the adiabatic compression. Consequently the flow pattern shown in Figs. 7 (a) and 8 (d) was formed. This flow pattern is dumping due to the classical viscosity in the right hand side of Eq. (1), because this shear flow is not so strong as to generate the Kelvin-Helmholtz instabilities, as seen in Fig. 7. The equilibrium quantities of $\hat{\rho}_{0}$ and $\hat{T}_{0}$ shown in Figs. 8 (b) and (c) have a flatter slope in the region $x \lesssim 0.5$ than those in $x \gtrsim 0.5$, which reminds us of the transport barrier observed in toroidal confinement systems. However, these radial profiles decrease radially, so that the system is unstable for the flute modes without counter shear flows. That is, Figs. 7 and 8 show that the counter shear flows prevent the flute modes from being unstable. 


\section{Summary}

We wrote a computer code using the basic equations obtained in Ref. [4]. These equations exclude the shear Alfvén modes and compressional modes from MHD equations in order to follow flute modes and the instability driven by nonuniform plasma flows (similar to the KelvinHelmholtz instabilities). We conducted the computer simulation in a modeled divertor mirror cell in the initial condition with finite rigid rotation but no shear flows.

Initial small temperature dip around the outer boundary causes flute instabilities. The flute instabilities are accompanied by large enhanced radial transport of mass density and temperature. At the end of the computer simulation, the counter shear flow appears, which suppresses the anomalous radial transport similar to the transport barrier formation. The equilibrium density and temperature with radially decreasing profiles continue to be stable under the existence of counter shear flows.

[1] I. Katanuma, Y. Sasagawa, Y. Tatematsu, Y. Nakashima, T. Cho and V.P. Pastukhov, Nucl. Fusion 46, 608 (2006).

[2] Y. Sasagawa, I. Katanuma, Y. Mizoguchi, T. Cho and V.P. Pastukhov, Phys. Plasmas 13, 122506 (2006).

[3] I. Katanuma, Y. Tatematsu, K. Ishii, T. Tamano and K. Yatsu, J. Phys. Soc. Jpn. 69, 3244 (2000).

[4] P.V. Pastukhov, Plasma Phys. Rep. 31, 577 (2005).

[5] S. I. Braginskii, Reviews of Plasma Physics, Vol. 1, Ed. M.A. Leontovich (Consultants Bureau, New York, 1965). 